\title{
Genetic relatedness among isolates of Acanthamoeba based on RAPD analysis
}

\begin{abstract}
Acanthamoeba is a pathogen frequently infecting brain, eyes, skin and lung of human and animal. Seven Acanthamoeba local isolates from various sources, two Acanthamoeba type strains and one strain of Hartmannella vermiformis were characterized for their genetic variability using randomly amplified polymorphic DNA (RAPD) technique using four different 10-mer oligonucleotides primers. Electrophoresis of the amplification products generated DNA bands ranging from approximately 0.25 to $7.50 \mathrm{kbp}$ in size. A genetic relatedness among the isolates was examined using Dice similarity coefficient as the genetic distance measured between the strains of Acanthamoeba and $\mathrm{H}$. vermiformis. Three distinct clusters could be separated at genetic distance of approximately 0.330. () 2006 Asian Network for Scientific Information.
\end{abstract}

Keyword: Acanthamoeba; DNA polymorphism; Genetic distance; RAPD-PCR 